\title{
Estudo Prospectivo da Tecnologia Intitulada "Barra com Esferas Virtuais e sua Aplicação em Testes de Desempenho de Máquinas de Medir por Coordenadas"
}

\author{
Virtual Spheres Bar and its Application in Performance Tests of \\ Measuring Machines by Coordinates
}

\author{
Krishna Aum Faria ${ }^{1}$ \\ Carlos Roberto Valeriano Junior ${ }^{1}$ \\ Iago Farias Lora, Raquel Santos ${ }^{1}$ \\ Paulo Gustavo ${ }^{1}$ \\ Barboni Dantas Nascimento ${ }^{1}$ \\ Grace Ferreira Ghesti ${ }^{1}$ \\ ${ }^{1}$ Universidade de Brasília, Brasília, DF, Brasil
}

\begin{abstract}
Resumo
O presente artigo realizou a prospecção tecnológica da tecnologia que foi protegida, intitulada "barra com esferas virtuais e sua aplicação em testes de desempenho de máquinas de medir por coordenadas", de titularidade da Fundação Universidade de Brasília. A análise buscou avaliar o potencial mercadológico da tecnologia em nível mundial, com buscas tanto em bases internacionais de patentes como em artigos. O país que mais depositou patentes foi a Rússia, em virtude de sua corrida espacial e de seus esforços militares. Quanto aos depositantes, percebeu-se uma maior concentração de empresas em detrimento de universidades e centros tecnológicos. Todavia, a quantidade de artigos publicados é muito mais significativa do que a de depósitos de patentes, inferindo-se que o tema abordado desperta mais interesse da academia do que da indústria. Por fim, constatou-se que a tecnologia se encontra obsoleta em relação a outras já comercializadas. Portanto, um contrato de transferência de tecnologia por meio de know how pode ser mais plausível e economicamente mais viável.
\end{abstract}

Palavras-chave: Máquinas de Medir por Coordenadas (MMC). Braço articulado. Calibração. Mensuração. Avaliação. Metrologia

\begin{abstract}
The present article seeks to reflect on the technological prospection of the patent "bar with virtual spheres and its application in performance tests of coordinate measuring machines" owned by University of Brasilia. The analysis seeks to evaluate the market potential of technology worldwide, with searches in both international patent bases and papers. The country that most deposited patents was Russia, because of its space race and its military efforts. As for the depositors, a greater concentration of companies was perceived, to the detriment of universities and technological centers. However, the number of articles published is much more significant than that of patent deposits, with the conclusion that the subject is more interested in academia than in industry. Finally, it was found that the technology is obsolete in relation to others already commercialized. Therefore, a contract for technology transfer through knowhow may be more plausible and economically more feasible.
\end{abstract}

Keywords: Coordinate Measuring Machines (MMC). Articulated arm. Calibration. Measurement. Evaluation. Metrology. Área Tecnológica: Instrumentação mecânica. Engenharia mecatrônica. 


\section{Introdução}

A engenharia mecatrônica aborda diversos ramos, dentre eles, os conceitos que permeiam as necessidades emergentes de integração e interação entre os diversos saberes da engenharia. Logo, fica evidente a sua relevância na execução de projetos, possibilitando, por sua vez, uma influência tanto no processo de desenvolvimento dos produtos (QUINTELLA et al., 2011) quanto na implementação de hodiernas técnicas no campo da engenharia. Dentre ferramentas contemporâneas da área supramencionada destaca-se a criação de produtos e processos que quando integrados são aplicados em cenário industrial e fundamentam e consolidam o avanço da tecnologia da informação.

As máquinas de medir por coordenadas como parte dos avanços tecnológicos no campo da engenharia determinam universalmente, por meio de dispositivos e instrumentos específicos, as posições de certos pontos sobre a peça a ser controlada. Tais pontos, quando processados pelo computador interligado, resultam nos parâmetros geométricos da peça controlada (CAVACO, 2002).

A relevância desse tipo de tecnologia é cada vez mais presente na garantia da qualidade dos produtos e a grande maioria das empresas de médio ou grande porte da área metal-mecânica possui máquinas utilizadas para determinar coordenadas nas suas linhas de metrologia. Ainda, por oportuno, estima-se que a indústria brasileira dispõe de mais de 6 (seis) mil equipamentos para medição por coordenadas, incluindo máquinas cartesianas e braços de medição (SOUSA, 2008). No entanto, as máquinas em evidência ainda possuem um alto custo de aquisição e manutenção. Desse modo, a indústria atuante na área persegue novos equipamentos e tecnologias que possam reduzir o custo do processo e consequentemente do produto (MELO et al., 2018).

Diante dos fatores externos, que não são considerados pela estrutura matemática dos computadores ligados à máquina ou por ela em si, é necessário um meio de correção ou integração com a equação realizada. Dessa maneira é possível recompor o sistema excluindo as variáveis externas (ou calibrando de maneira a considerar as circunstâncias). Por meio da calibração, as imperfeições são balanceadas com o posicionamento da máquina, podendo assim, corrigir as variações e imprecisões (PIRATELLI-FILHO, 2010; MELO et al., 2018).

Nesse contexto, a tecnologia objeto central do presente estudo consiste na patente de invenção intitulada "barra com esferas virtuais e sua aplicação em testes de desempenho de máquinas de medir por coordenadas" de titularidade da Fundação Universidade de Brasília. Vale dizer que a tecnologia em comento se refere a um instrumento utilizado como padrão para calibração ou para testes de verificação de desempenho de Máquinas de Medir por Coordenadas (MMC), principalmente para os modelos de braço articulado, em substituição ao modelo de barras com esferas e barras de esfera (PIRATELLI-FILHO, 2010). O método de uso do padrão está detalhado na norma americana ANSI/ASME 889.1.12, elaborada para a aplicação da barra de esferas.

Assim, por meio do presente artigo busca-se a realização de um estudo prospectivo acerca do cenário mundial da tecnologia em evidência, no intuito de se identificar o nível de maturidade da invenção, o nicho tecnológico mais adequado para o seu lançamento no mercado, $e$ a relação entre documentos patentários e artigos publicados em revistas indexadas. 


\section{Método}

O método de pesquisa empregado na consecução do presente trabalho avalia instrumentos utilizados para a realização de teste de medição e calibragem de máquinas industriais, no cenário mundial. Para tanto, foram utilizadas bases internacionais de patentes e de artigos publicados em revistas indexadas (Orbit e Web Of Science).

As buscas foram realizadas mediante o emprego de várias combinações de palavras-chave, nos campos "título" e "resumo", delimitando-se o período de tempo compreendido entre janeiro de 2008 até dezembro de 2018. Desse modo, foram utilizadas especificamente as seguintes palavras-chave: calibration, measuring e instrument. Os tradicionais operadores booleanos (and e or), técnicas de truncamento "(*)" e a associação de sinônimos para as palavras-chave foram utilizados para composição do método de busca da pesquisa.

Por meio dos métodos acima descritos, foram identificadas as principais classificações de (IPC), os países detentores dos maiores indicadores de prioridade, bem como as empresas com os maiores números de depósitos de patentes. Técnica que possibilitou a análise comparativa entre a evolução do número de proteções no decorrer dos últimos 10 (dez) anos e o número de publicações científicas inerentes ao tema estudado em igual período.

Quadro 1 - Argumentos de booleanos utilizados no Patentscope

\begin{tabular}{|c|}
\hline CALIBRATION AND MEASURING AND INSTRUMENT \\
CALIBRAT* AND MEASUR* AND INSTRUMENT \\
\hline (CALIBRAT* OR EVALUAT*) AND MEASUR* AND INSTRUMENT \\
\hline (CALIBRAT* OR EVALUAT*) AND MEASUR* AND INSTRUMENT* AND METROLOG* \\
\hline
\end{tabular}

Fonte: Elaborado pelos autores deste artigo

Quadro 2 - Argumentos de booleano utilizado no Web of Science por combinações de palavras-chaves com delimitação de período determinada entre 2008 a 2018

(CALIBRAT* OR EVALUAT*) AND MEASUR* AND INSTRUMENT* AND METROLOG*

Fonte: Elaborado pelos autores deste artigo

\section{Resultados e Discussão}

A busca pela combinação das palavras-chaves "calibration" "measuring" e "instrument" utilizando o operador booleano "AND" retornou 1.882 (mil oitocentos e oitenta e dois) resultados. O sinônimo da palavra calibration, qual seja, "evaluat", obteve como repercussão 2.813 (dois mil oitocentos e treze) respostas.

Seguindo um refinamento da busca, foi acrescentado ao final da combinação de palavras-chaves o termo "metrolog*", que resultou na limitação dos dados de enquadramento da tecnologia explorada a 30 (trinta) respostas. 
Quadro 3 - Resultado da busca de pedidos de patentes, na base de dados Patenscope, por combinações de palavras-chaves com delimitação de período determinada entre 2008 a 2018

\begin{tabular}{|c|c|}
\hline ARGUMENTO DE PESQUISA & No DE DOCUMENTOS ENCONTRADOS \\
\hline CALIBRATION AND MEASURING AND INSTRUMENT & 1.882 \\
\hline CALIBRAT* AND MEASUR* AND INSTRUMENT & 1.881 \\
\hline $\begin{array}{c}\text { (CALIBRAT* OR EVALUAT*) AND } \\
\text { MEASUR* AND INSTRUMENT }\end{array}$ & 2.813 \\
\hline $\begin{array}{c}\text { (CALIBRAT* OR EVALUAT*) AND MEASUR* } \\
\text { AND INSTRUMENT* AND METROLOG* }\end{array}$ & 30 \\
\hline
\end{tabular}

Fonte: Elaborado pelos autores deste artigo

Quadro 4 - Resultado da busca de publicações científicas na base de dados Web of Science, por combinações de palavras-chaves com delimitação de período determinada entre 2008 a 2018

\begin{tabular}{|c|c|}
\hline ARGUMENTO DE PESQUISA & TOTAL \\
\hline \begin{tabular}{c|c|} 
(CALIBRAT* OR EVALUAT*) AND MEASUR* \\
AND INSTRUMENT* AND METROLOG*
\end{tabular} & 911 \\
\hline
\end{tabular}

Fonte: Elaborado pelos autores deste artigo

Desse modo, partindo-se do parâmetro de 30 documentos patentários, resultado do último refinamento de pesquisa, foram realizadas as análises dos principais IPCs, países com maior número de prioridade, principais empresas depositantes, e a comparação dos números de documentos patentários em relação aos números de artigos publicados em revistas indexadas ao longo dos últimos 10 (dez) anos, a fim de mensurar a maturidade da tecnologia.

A partir do refinamento para identificar a Classificação Internacional de Patentes (IPC), foi encontrado um número considerável de variações de subclasses, totalizando 10 subclassificações mais utilizadas. Conforme o Gráfico 1, exposto a seguir, o maior número concentrou-se na subclasse $\mathrm{G} 01 \mathrm{~B}$, quantificando 07 publicações, cujo teor está relacionado com a medição de comprimentos, espessura ou outras dimensões lineares semelhantes, medição de ângulos, medição de áreas, medição de irregularidades de superfícies ou contornos. Logo em seguida, vieram as subclasses G01C e G01N, totalizando 06 publicações, sendo estas relacionadas com a medição de distâncias, níveis ou rumos, topografia, navegação, instrumentos giroscópicos, fotogrametria ou videogrametria; e com a investigação ou análise dos materiais pela determinação de suas propriedades químicas ou físicas, respectivamente. Por fim, com o menor número de publicações, observaram-se as subclasses A61B, B23Q, G01M, G01W, G06F, G01Ke G01L. A subclasse A61B está relacionada com diagnóstico, cirurgia, identificação; a subclasse B23Q tem relação com detalhes, peças ou acessórios de máquinas-ferramenta, por exemplo, disposições para reprodução ou controle; (ferramentas de tipo empregado em tornos ou máquinas de broquear B23B 27/00) máquinas-ferramenta em geral, caracterizadas pela estrutura de detalhas ou peças especiais, combinações ou associações de máquinas, para a usinagem de metal, não destinadas a um resultado específico. A subclasse G01M envolve o teste do equilíbrio estático ou dinâmico de máquinas ou estruturas, teste de estruturas ou de aparelhos não incluídos em outro local. A subclasse G01W tem relação com meteorologia, envolvendo radar, sonar, LIDAR ("Light Detection and Ranging") ou sistemas análogos desenvolvidos para uso meteorológico. A G06F diz respeito ao processamento elétrico de dados digitais (sistemas 
de dados baseados em modelos computacionais específicos G06N). A G01K trata da medição de temperatura, medição da quantidade de calor, elementos termossensíveis não incluídos em outro local (pirometria das radiações G01J 5/00). Por fim, a subclasse G01L está relacionada com a medição da força, tensão, torque, trabalho, potência mecânica, eficiência mecânica, ou pressão dos fluidos (pesagem G01G).

Gráfico 1 - Número de depósitos em função da Classificação Internacional de Patentes (IPC) período entre 2008 a 2018

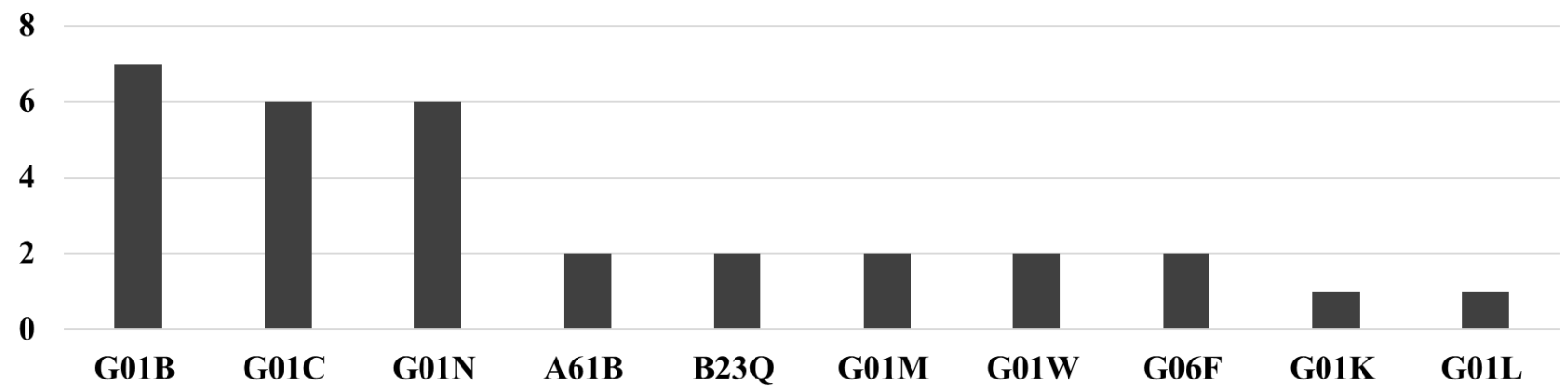

Fonte: Elaborado pelos autores deste artigo

O Gráfico 2, abaixo representado, mostra dados dos principais países depositantes da tecnologia estudada em contexto global. Neste sentido, observa-se que a Federação Russa predominou quanto ao número de depósitos, totalizando 16 depósitos. Seguindo a Federação Russa aparece a China - 4 depósitos; 2 no European Patent Office (EPO); 2 nos Estados Unidos da América; 1 no Reino Unido; e 1 em Portugal.

Gráfico 2 - Resultados em razão dos principais países de prioridade no período de 2008 a 2018

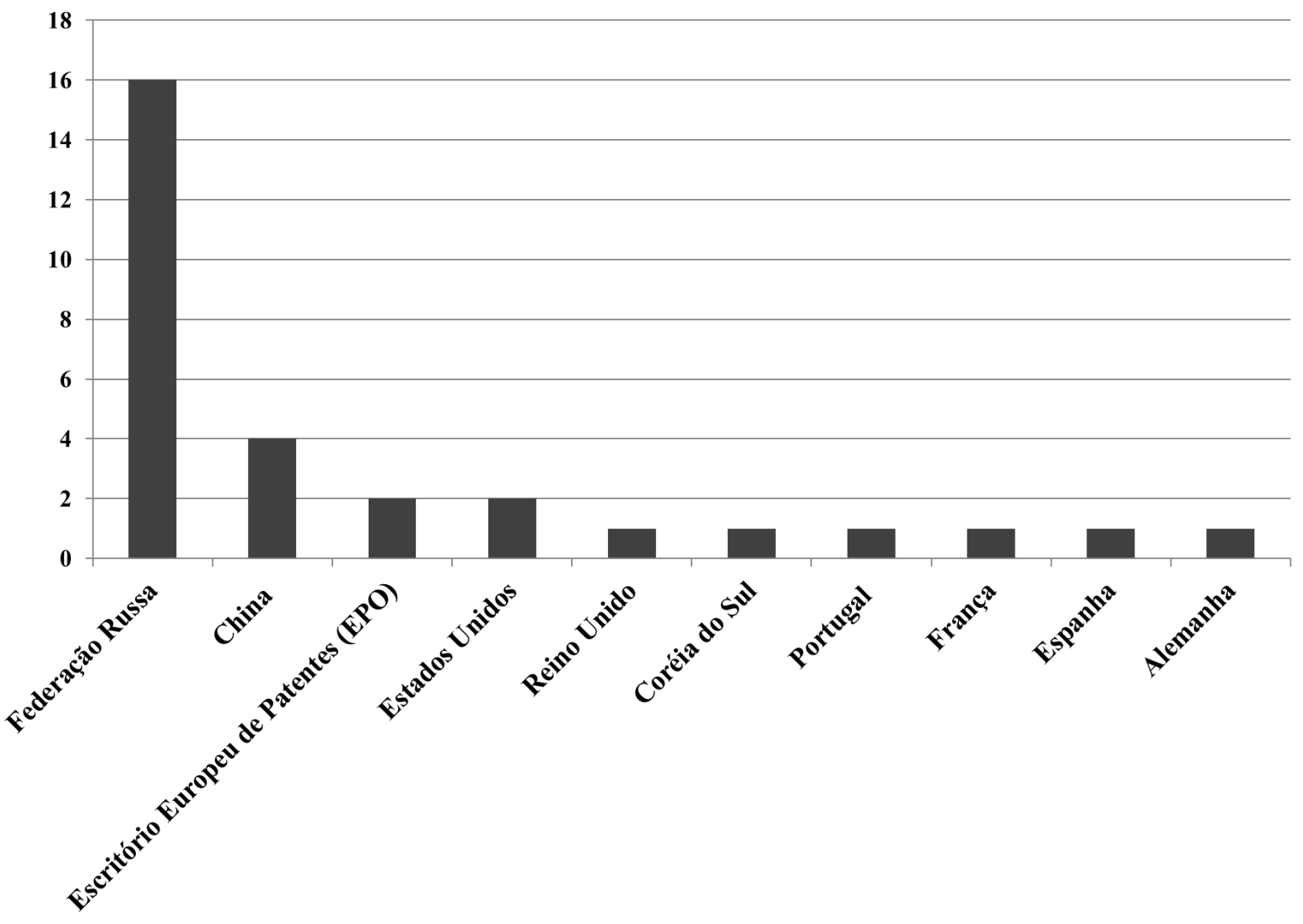

Fonte: Elaborado pelos autores deste artigo 
Vale dizer que o interesse russo no desenvolvimento desse tipo de tecnologia vem de períodos pretéritos, antes da própria “[...] Revolução Russa de 1917, quando surgiram grupos ligados à cosmologia, que acreditavam ser fundamental difundir ideias sobre o Espaço e sobre viagens planetárias para o grande público" (BORGES, 2013, p. 72). Um programa que foi idealizado com o condão de pendor militar e secreto, por fim, foi responsável por grandes avanços da humanidade: como o primeiro míssil balístico intercontinental, o primeiro satélite artificial, o primeiro animal no espaço, o primeiro homem no espaço, ou ainda, o primeiro satélite artificial na lua (FRAZÃO, 2015). Assim, foi observada ao longo da história recente da Federação Russa, uma estratégia consistente e produtiva, direcionada ao desenvolvimento de tecnologias no campo da engenharia espacial/mecatrônica, o que justifica a liderança desse país no depósito de patentes desse segmento.

O Gráfico 3 possibilita evidenciar a predominância de empresas em detrimento das universidades e centros tecnológicos. Ou seja, as patentes foram depositadas visando à comercialização a fim de garantir exclusividade e retorno à empresa depositante. No entanto, verifica-se que as empresas Phasics e Taylor Hobson Ltd são as maiores detentoras de pedidos já publicados.

Gráfico 3 - Resultado dos principais depositantes entre o período de 2008 a 2018

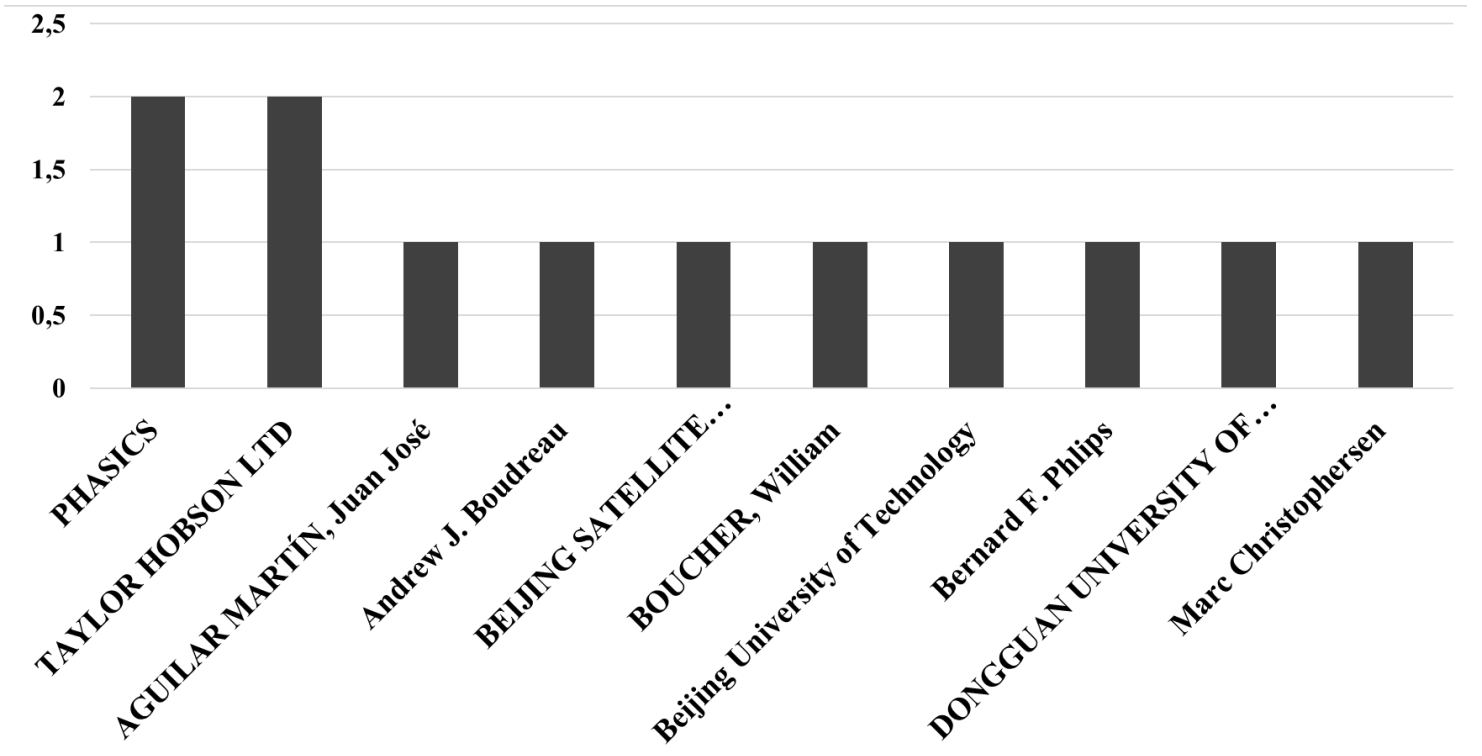

Fonte: Elaborado pelos autores deste artigo

A empresa Phasics, por exemplo, é especializada em sensores de frente de onda de alta resolução e oferece uma linha robusta de analisadores Wave front de alto desempenho e soluções integradas de metrologia, conforme informações constantes no sítio eletrônico da própria empresa. No mesmo sentido, a Taylor Hobson Ltd é uma empresa de tecnologia de ultraprecisão operando no campo da metrologia de superfície e formas.

Nesse sentido, é possível verificar que empresas que desenvolvem produtos, ou processos ligados à área de metrologia, são potenciais interessadas em tecnologias semelhantes ao produto objeto central do presente estudo. Porém, a patente foi depositada no Brasil e não nos demais países em que essas empresas atuam. Dessa forma, a comercialização não é viável para empresas de outros países em função do baixo grau de maturidade e pelo fato de a tecnologia estar obsoleta. 
Para o intervalo temporal - entre os anos (2008-2018) foram identificados 30 (trinta) documentos patentários. Inicialmente, o período correspondente ao ano de 2008 indica baixo interesse pela proteção. Em contrapartida, observou-se que entre os anos de 2009 a 2013 houve uma evolução com um maior número de interesse no registro, totalizando 19 (dezenove) documentos publicados nesse período. A partir daí, verificou-se um expressivo decréscimo no interesse pela proteção da patente, a contar do ano de 2014 até o ano de 2018, totalizando 09 (nove) documentos patentários, havendo, portanto, uma redução de aproximadamente $50 \%$ no interesse pela proteção.

No que tange à publicação de artigos científicos no cenário mundial, foram obtidos 911 (novecentos e onze) entre os anos de 2008 e 2017, utilizando-se as palavras-chaves "(calibrat* orevaluat*) andmeasur* andinstrument* andmetrolog*", conforme se verifica na Tabela 2. Observou-se que nos primeiros 07 (sete) anos, entre 2008 e 2014, houve predominância de uma média constante, sem diferenças quantitativas entre os registros a cada ano. Já entre os anos de 2015 e 2016, foi publicada a maior concentração de artigos, o que demonstra que, estatisticamente, a quantidade de publicações de artigos científicos vem crescendo consideravelmente, apesar da queda registrada no ano de 2017.

Quadro 5 - Comparação - Ano, Artigos e Patentes

\begin{tabular}{|c|c|c|}
\hline ANO & ARTIGOS & PATENTES \\
\hline 2008 & 69 & 2 \\
\hline 2009 & 76 & 6 \\
\hline 2010 & 78 & 4 \\
\hline 2011 & 86 & 3 \\
\hline 2012 & 95 & 1 \\
\hline 2013 & 88 & 5 \\
\hline 2015 & 94 & 2 \\
\hline 2016 & 130 & 2 \\
\hline 217 & 105 & 4 \\
\hline
\end{tabular}

Fonte: Elaborado pelos autores deste artigo

Analisando-se o Gráfico 4, a dispersão do número de documentos patentários em relação ao número de produções científicas ao longo dos anos de 2008 a 2018, fica evidente que o número de artigos publicados ultrapassa de forma exponencial a produção patentária ao longo de todos os anos analisados. Infere-se, da presente análise, que embora exista algum movimento da indústria em proteger ativos relacionados a instrumentos de medição, o tema em questão ainda flutua sobre a esfera de produção acadêmica, o que justifica a baixa maturidade tecnológica. 
Gráfico 4 - Análise por combinação entre documentos patentários e artigos científicos no período de 2008 a 2018 (gráfico de coluna 100\% empilhada)

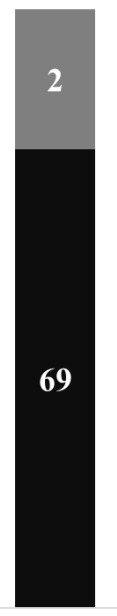

2008

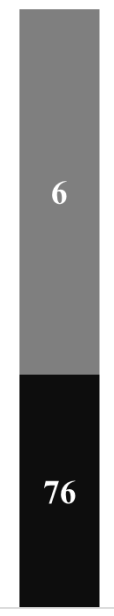

2009

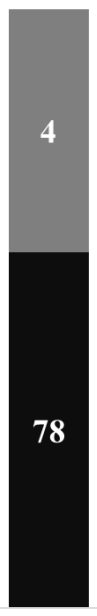

2010

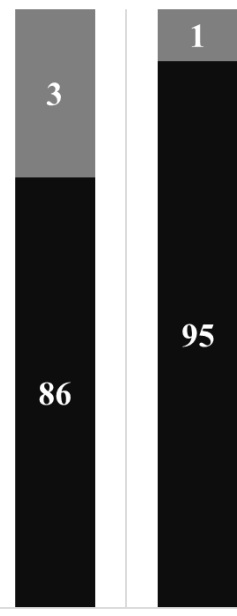

2011

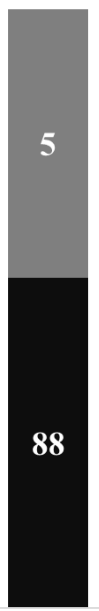

2013

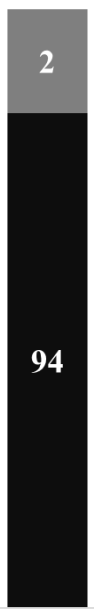

2014

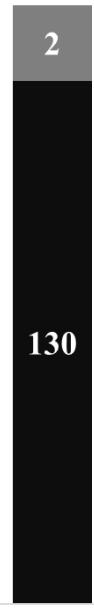

2015

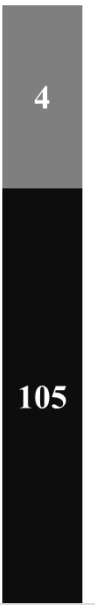

2016

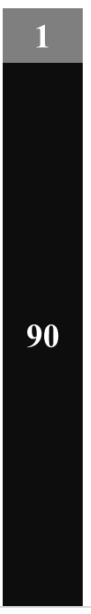

2017

- Artigos $\square$ Patentes

Fonte: Elaborado pelos autores deste artigo

Desse modo, pode-se afirmar que a maturidade da tecnologia estudada ainda é baixa, principalmente se for levado em consideração que ao longo dos últimos anos a publicação de artigos científicos ligados ao tema tem crescido de forma evidente.

O modelo construído pela equipe de pesquisadores foi submetido a diversos testes no Laboratório de Metrologia da Universidade de Brasília, demonstrando resultados satisfatórios nas medições de padrões e calibração de máquinas de medir por coordenadas de braço articulado, permitindo que o invento se estabeleça entre o TRL níveis 3 e 4.

A presente tecnologia, como abordado no decorrer do texto, necessita de investimento para que ocorra o desenvolvimento tecnológico e haja aumento do grau de maturidade. Atualmente, a tecnologia se encontra obsoleta comparando-a com outras já comercializadas, porém alguns pontos podem ser desenvolvidos nos modelos comerciais. Sendo assim, um contrato de transferência de tecnologia por meio de know how é possível, aperfeiçoando os modelos já existentes. O Quadro 5 traz o perfil de 3 (três) empresas (possíveis concorrentes tecnológicos), mas que poderiam também serem acionadas a fim de elaborarem um projeto de desenvolvimento tecnológico conjunto.

Quadro 5 - Possíveis concorrentes

\begin{tabular}{|c|c|}
\hline PHASICS & $\begin{array}{l}\text { Com ampla atuação conjunta com o setor acadêmico, a empresa } \\
\text { Phasics, sediada em Saint-Aubin - França, oferece solução de } \\
\text { medição por laser, microscópios e lentes. Devido a seu tempo de } \\
\text { mercado, pode ser considerada uma companhia já consolidada. }\end{array}$ \\
\hline TAYLOR HOBSON LTD & $\begin{array}{l}\text { Fundada em } 1886 \text { a companhia é de grande porte, Taylor Hobson } \\
\text { LTD tem sede na Inglaterra, atua na área de bens de medição por } \\
\text { contato ou sem contato. É forte no mercado de desenvolvimento de } \\
\text { tecnologias utilizadas pelo setor automotivo, médico e aeroespacial. }\end{array}$ \\
\hline GHM GROUP & $\begin{array}{l}\text { O GHM Group, fundado em 1963, possui atuação internacional } \\
\text { (incluindo o território brasileiro), atua na indústria automobilística, } \\
\text { setor de energia, automação predial e fabricação de máquinas. }\end{array}$ \\
\hline
\end{tabular}

Fonte: Elaborado pelos autores deste artigo 


\section{Considerações Finais}

O estudo de prospecção tecnológica resultou em 30 (trinta) documentos patentários e 911 (novecentos e onze) artigos publicados. A análise dos resultados permitiu identificar um pico de publicações nos anos 2009 (6) e 2013 (5). Já da análise dos artigos científicos, percebe-se que a curva foi ascendente ao longo dos anos utilizados como recorte temporal, alcançando certa estabilidade nos anos de 2016 e 2017.

Conclui-se que o expressivo número de publicações científicas triunfa sobre o quantitativo de proteção patentária; o que demonstra que a rota tecnológica a qual se insere a tecnologia estudada ainda é baixa e que o conhecimento e o desenvolvimento permanecem de forma quase predominante na academia.

Além do mais, conclui-se que a Rússia se destaca como o país de prioridade em detrimento dos demais países depositantes, figurando, dessa forma, como o grande centro de desenvolvimento de tecnologias ligadas ao objeto cerne deste estudo.

Por fim, conclui-se que do potencial tecnológico da invenção e das vantagens em relação às demais tecnologias utilizadas, o número amplamente superior de publicações científicas demonstra que ainda existe um vasto campo de possibilidades para pesquisa e desenvolvimento nesta área.

\section{Referências}

BORGES, Fabiane Morais. Na busca da cultura espacial. 2013. 207f. Tese (Doutorado em Psicologia Clínica - Estudos da Subjetividade) - Pontífica Universidade Católica de São Paulo. São Paulo, 2013.

CAVACO, Marco Antônio Martins. Metrologia: Parte 2. [2002]. Disponível em: http://www.ifomep. org.br/ava/cursos/aperfeicoamento/controle_dimensional/apoio1.pdf. Acesso em: 27 abr. 2018.

FRAZÃO, João Nuno Fernandes. A regulação da atividade espacial: a questão emergente da responsabilidade civil no transporte turístico aeroespacial. 2015. 95f. Dissertação (Mestrado em Direito) - Faculdade de Direito da Universidade Nova de Lisboa. Lisboa, 2015.

MELO, J. dos S. et al. Utilização de ferramentas de inteligência competitiva para delinear estratégias de posicionamento de mercado de equipamentos eletromédicos de monitoramento. Cader.

Prospec., Salvador, v. 11, Edição Especial, p. 211-224, abr.-jun., 2018. [DOI: http://dx.doi. org/10.9771/cp.v11i2.23019].

PIRATELLI-FILHO, Antônio. Barra com esferas virtuais e sua aplicação em testes de desempenho de máquinas de medir por coordenadas. Int. Cl: G01B 21/20. BRn. PI0803145-2 A2. 02. Mar. 2010.

QUINTELLA, C. M. et.al. Prospecção Tecnológica como uma Ferramenta Aplicada em Ciência e Tecnologia para se Chegar à Inovação. Rev. Virtual Quim., São Paulo, v. 3, n. 5, p. 406-415, 2011.

SOUSA, André Roberto. Importância de formação metrológica avançada na área de medição por coordenadas na indústria. [2008]. Disponível em: http://www.forma3d.com.br/downloads/ Importancia\%20da\%20formacao\%20de\%20metrologistas.pdf. Acesso em: 17 jun. 2018. 


\section{Sobre os Autores}

\section{Krishna Aum Faria}

E-mail:krishna13@uol.com.br

Mestrando em Propriedade Intelectual e Transferência de Tecnologia para a Inovação pela Universidade de Brasília. Endereço profissional: SGAS Quadra 605, Conjunto A, Asa Sul, Brasília, DF. CEP: 70200-904.

\section{Carlos Roberto Valeriano Junior}

E-mail: cvaleriano89@gmail.com

Mestre em Propriedade Intelectual e Transferência de Tecnologia para a Inovação pela Universidade de Brasília (2019).

Endereço profissional: Campus Universitário Darcy Ribeiro, Av. L3 Norte, Ed. Finatec, Asa Norte, Brasília ,DF.

\section{Iago Farias Lora}

E-mail: iagolora@outlook.com

Mestrando em Propriedade Intelectual e Transferência de Tecnologia pela Universidade de Brasília - UNB/PROFNIT.

Endereço profissional: SHIS QI 26, conjunto 2, casa 26, Lago Sul, Brasília, DF. CEP: 71670-020.

\section{Raquel Santos}

E-mail: raquelsanto@gmail.com

Mestranda em Propriedade Intelectual e Transferência de Tecnologia e Inovação pela Universidade de Brasília. Endereço profissional: Praça dos Três Poderes - Brasília, DF. CEP: 70175-900.

\section{Paulo Gustavo Barboni Dantas Nascimento}

E-mail:pgbdantas@gmail.com

Doutor em Química na FCFRP - Universidade de São Paulo (USP) (2005). Pós-Doutor em Farmacologia pela FMRP (2009).

Endereço profissional: Universidade de Brasília, UNB - Campus Ceilândia. QNN14 - Área Especial. Ceilândia Sul, Brasília, DF, Brasil. CEP: 72220-140.

\section{Grace Ferreira Ghesti}

E-mail: grace@unb.br

Doutora em Química pela Universidade de Brasília (2009).

Endereço profissional: Universidade de Brasília, Instituto de Química. Campus Universitário Darcy Ribeiro, Instituto de Química, Laboratório B1-75/31, Asa Norte, Brasília, DF, Brasil. CEP: 70904-970. 